\title{
Robust Image Watermark Using Radon Transform and Bispectrum Invariants
}

\author{
Hyung-Shin Kim ${ }^{1}$, Yunju Baek ${ }^{1}$, Heung-Kyu Lee ${ }^{1}$, and Young-Ho Suh ${ }^{2}$ \\ 1 Division of Computer Science \\ Department of Electrical Engineering \& Computer Science \\ Korea Advanced Institute of Science and Technology \\ 373-1 Kusung-Dong Yusong-Gu Taejon, 305-701, South Korea \\ hskim@casaturn.kaist.ac.kr \\ 2 Contents Technology Department \\ Electronics and Telecommunications Research Institute(ETRI) \\ 161 Gajeong-Dong, Yuseong-Gu, Daejeon, 305-350, South Korea
}

\begin{abstract}
Image watermark that is resistant to geometric distortion is remained to be an unsolved problem. Difficulty of the probelm comes from the situation that the watermark should be extracted without any information of the original image. In this paper, we review this problem and propose a new watermarking scheme based on invariant pattern recognition theory. We propose an invariant watermark using the Radon transform and higher order spectra. A bispectrum feature vector of the image is used as the watermark. Our approach differs from the previous methods in that we embed watermark into the phase of the higher order spectra. Also, our Radon embedding grid outperforms the Fourier-Mellin based methods. We devised a new embedding method which allows detection of the watermark when there is no exact inverse function during embedding. As we use the Radon transform, our method can be used for medical images. We show the invariance of the designed watermark with mathematical proofs. Experimental results confirm that this scheme is resistant to geometric distortions.
\end{abstract}

\section{Introduction}

There has been a very intensive research in the digital watermarking area in the last few years [1]. A useful image watermarking method must be robust to the distortions occurred by any normal use of images. Those distortions include a wide range of image processing such as image enhancement, JPEG compression and geometrical modifications. However, conventional image watermarking algorithms are sensitive to geometric distortions [2]. Simple rotation, scale and translation may significantly reduce the detection level since it changes the alignment of the watermark. Random geometric distortion which is known as StirMark attack [2] greatly reduces the watermark strength at the detector.

Some watermarking methods that are resilient to geometrical attacks were reported in recent papers. One approach is to embed a known template into 
images along with the watermark [3][4]. The template contains the information of the geometric transform undergone by the image. During detection, the image is inverse transformed using the distortion information estimated from the template, then the watermark can be extracted. This method requires embedding a template in addition to the watermark so that this may reduce image fidelity and watermark capacity. Watermarks itself can be used as a template [5]. They embed a signal multiple times and use autocorrelation for the detection of the watermark pattern. The autocorrelation peaks have the structure for inverting geometric distortion. For this type of system to be robust, both the inverting and watermark detection procedure should be robust.

Another approach is to insert a watermark in a domain that is invariant to geometrical distortions based on the Fourier-Mellin transform [6]. A rotation and scaling of an image results in the translation at the log-polar mapping of the Fourier magnitude spectrum of the image. After taking the Fourier magnitude spectrum of the modulus of it, we reach to the RST invariant domain. Watermark is inserted in the domain and inverted to have the watermarked image. One problem with this method is that they have implementation difficulty. The log-polar and inverse log-polar mapping process uses interpolation that causes a degradation of the watermark and fidelity loss of the watermarked image. The second problem is in that they need the original image for the watermark detection. Any geometric distortion can be inverted with the original image.

Watermarks that are invariant to geometric distortions can be designed [7]. They use the Fourier-Mellin transform for the required invariance. They define an invariant vector from the central slices of the log-polar mapped Fourier magnitude spectrum. A watermark is embedded by modifying the vector. Algorithms using the Fourier-Mellin transform suffer serious implementation difficulties. The log-polar and inverse log-polar mapping introduces errors during insertion. As they use the Fourier magnitude spectrum, interpolation performance is poor because interpolation only performs well with the sample values with the same scale. There are many other implementation problems to consider [6][7].

Watermarking algorithms using a feature of an image were proposed as the second generation watermark [8][9]. As features of the image have high invariance to distortions, they can be used as a key to find the insertion location. We propose a feature based image watermarking method that is resistant to geometrical attacks. An invariant feature vector is defined with higher order spectra (HOS) of an image. The HOS were introduced as spectral representations of cumulants or moments of ergodic processes, and are useful in the identification of nonlinear and non-Gaussian random processes as well as deterministic signals [10][11]. The use of HOS for our feature-based watermark is motivated as HOS are translation invariant and they are zero for Gaussian noise.

For the use of HOS, we adopt the bispectrum (the third-order spectra) feature which is known to have a wide range of invariance properties. Invariant bispectrum features have been used in pattern recognition for texture analysis and image classification [12][13]. We define a bispectrum vector from the projections of an image. The vector is modified and inverted to have the watermarked 
image. However, due to its lossy nature of the inverse process, we can not detect the modified vector after the inversion.

As argued in [7], we believe that the strong invariance of the embedding function is not necessary. We can formulate this as follows. With an embedding function, $Y(w, C)$, we generate the watermarked image $C_{w}$ from the cover image $C$ and the watermark $w$. Instead of detecting the inserted signal $w$ from $C_{w}$, the detector $X\left(C_{w}\right)$ extracts the signal mixed with the watermarking system distortion $n_{s}$ as follows:

$$
X\left(C_{w}\right)=w^{\prime}=w+n_{s}
$$

If the function $Y$ can generate inverted image exactly during the embedding procedure, we have $n_{s}=0$ and we can extract the exact embedded watermark. However, as in [6] and [7], when we use an embedding function that has lossy inverse procedure, $n_{s}$ is not zero. These inversion losses occur when we apply the continuous functions to discrete images. In these situations, we still have to be able to detect $w$ from the distorted $w^{\prime}$. This problem can be solved in several approaches. By defining a function that maps $w$ from $w^{\prime}$, the embedded watermark can be extracted. Another way to avoid this problem is to design an inversion procedure that can minimize $n_{s}$.

These approaches generally introduce complexity to the system with high cost. We propose a low cost method using the detector information during the insertion procedure. We use a form of informed embedding of watermarking with side information [14]. First, we design a detector that extracts the feature vector from the projections of the test image. During the embedding procedure, the detector is called to estimate $w^{\prime}$. If $w^{\prime}$ has a detection value significantly greater than values for previously defined watermarks, it is accepted as the watermark and it is expected to be extracted at the detector instead of $w$. We repeat this procedure with different watermarks until we are successful. Watermarks are generated through this iterative routine to guarantee their uniqueness at the detector.

Our approach is similar to [7]. We define a vector from the projections. However, our method is different from them in that we use the bispectrum feature vector which has a wide range of invariance properties. We devised a new insertion method which does not require exact inversion process. Our method is more resilient to tampering as we embed a signal into the Fourier phase spectrum while the previous methods use the magnitude spectrum. This is because the phase components of the discrete Fourier transform (DFT) have more psychovisual impact than magnitude spectrum and as a consequence, malicious parties would need to cause unacceptable damage to the quality of the image in order to remove the watermark [15].

The proposed method is evaluated using the StirMark [2] benchmark software. The experimental results show that our algorithm performs well against the geometric distortions and other signal attacks.

The rest of this paper is organized as follows: Section 2 describes the bispectrum feature of images; Section 3 presents the watermarking algorithm; Section 4 


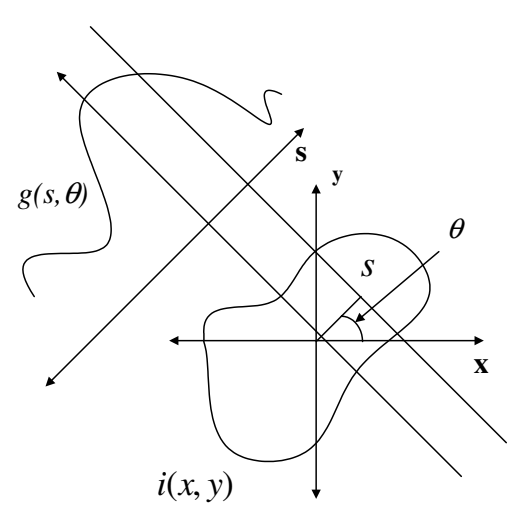

(a)

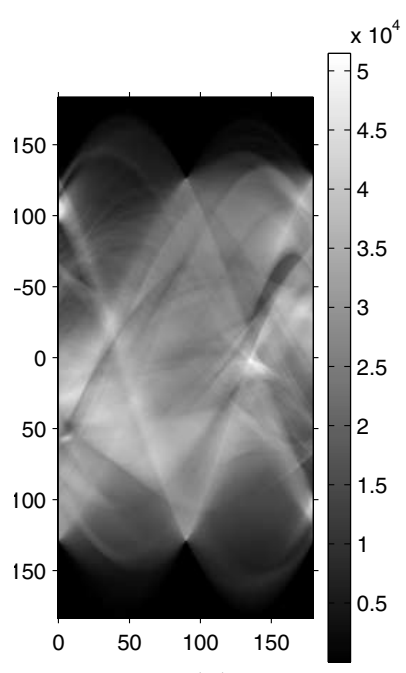

(b)

Fig. 1. Image decomposition with the Radon transform : (a) Projection process (b) 1-D Projection of Lena image

shows the experimental results of the proposed method; In section 5, we conclude with the contribution of our approach and directions for future development.

\section{Bispectrum Feature Vector of Images}

The bispectrum, $B\left(f_{1}, f_{2}\right)$, of a $1-\mathrm{D}$ deterministic real-valued sequence is defined as

$$
B\left(f_{1}, f_{2}\right)=X\left(f_{1}\right) X\left(f_{2}\right) X^{*}\left(f_{1}+f_{2}\right)
$$

where $X(f)$ is the discrete-time Fourier transform of the sequence $x(n)$ at the normalized frequency $f$. By virtue of its symmetry properties, the bispectra of a real signal is uniquely defined in the triangular region of computation, $0 \leq f_{2} \leq f_{1} \leq f_{1}+f_{2} \leq 1$. A 2 -D image is decomposed into $N 1$-D sequences $g(s, \theta)$ using the Radon transform. The Radon transform $g(s, \theta)$ of a 2-D image $i(x, y)$ is defined as its line integral along a line inclined at an angle $\theta$ from the $\mathrm{y}$-axis and at a distance $\mathrm{s}$ from the origin. This projection process and the Radon transform of Lena image is shown in Fig. 1.

The projection slice theorem [16] states that the Fourier transform of the projection of an image on to a line is the 2-D Fourier transform of the image evaluated along a radial line. From the theorem, we can use 2-D Fourier transform instead of the Radon transform during implementation. 
A parameter $p(\theta)$ is defined as the phase of the integrated bispectra of a 1-D Radon projection $g(s, \theta)$ along the line of $f_{1}=f_{2}$ and it can be expressed with the polar mapped 2-D DFT as follows using the projection slice theorem:

$$
\begin{aligned}
p(\theta) & =L\left[\int_{f_{1}=0^{+}}^{0.5} B\left(f_{1}, f_{1}\right) d f_{1}\right] \\
& =L\left[\int_{f_{1}=0^{+}}^{0.5} I^{2}(f, \theta) I^{*}(2 f, \theta) d f\right]
\end{aligned}
$$

Though the parameter can be defined along a radial line of slope $a, 0<a \leq 1$ in the bifrequency space, we compute $p(\theta)$ at $a=1$, where $f_{1}=f_{2}$. In this way, we can avoid interpolation during the computation of $p(\theta)$.

A vector $\boldsymbol{p}$ of length $N$ is defined as $\boldsymbol{p}=\left(p\left(\theta_{1}\right), p\left(\theta_{2}\right), \ldots, p\left(\theta_{N}\right)\right)$. From the properties of the Radon transform and bispectrum parameter $p(\theta), \boldsymbol{p}$ is invariant to dc-level shift, amplification, translation, scaling, and Gaussian noise [12]. As a rotation of the 2-D image results in a cyclic shift in the set of projections, $\boldsymbol{p}$ will be cyclically shifted as well. In [12], $\boldsymbol{p}$ was used as a feature for object recognition. We show the invariance properties of $\boldsymbol{p}$ against rotation, scaling, and translation in the following.

\subsection{Translation}

A translated version of an image $i(x, y)$ is represented as

$$
i^{\prime}(x, y)=i\left(x+x_{0}, y+y_{0}\right)
$$

The Fourier transform of $i^{\prime}(x, y)$ is $I^{\prime}\left(f_{x}, f_{y}\right)$ and it is given by

$$
I^{\prime}\left(f_{x}, f_{y}\right)=I\left(f_{x}, f_{y}\right) \cdot e^{j x_{0} f_{x}} \cdot e^{j y_{0} f_{y}}
$$

If we now rewrite (4) using polar coordinates,

$$
\begin{aligned}
& f_{x}=f \cos \theta \\
& f_{y}=f \sin \theta
\end{aligned}
$$

then (5) becomes,

$$
I^{\prime}(f, \theta)=I(f, \theta) \cdot e^{j x_{0} f \cos \theta} \cdot e^{j y_{0} f \sin \theta}
$$

From (3), the parameter $p^{\prime}(\theta)$ becomes,

$$
\begin{aligned}
& p^{\prime}(\theta) \\
& =L\left[\int_{0^{+}}^{0.5} I^{2}(f, \theta) I^{*}(2 f, \theta) e^{j\left(2 x_{0} f \cos \theta+2 y_{0} f \sin \theta-2 x_{0} f \cos \theta-2 y_{0} f \sin \theta\right)} d f\right]
\end{aligned}
$$




$$
\begin{aligned}
& =\angle\left[\int_{0^{+}}^{0.5} I^{2}(f, \theta) I^{*}(2 f, \theta) d f\right] \\
& =p(\theta)
\end{aligned}
$$

As (9) shows that $p(\theta)$ is invariant to translation, $\boldsymbol{p}$ is invariant to translation.

\subsection{Scaling}

A scaled image $i^{\prime}(x, y)$ can be expressed as

$$
i^{\prime}(x, y)=i(s x, s y)
$$

where $s$ is a scale factor. The polar mapped Fourier transform of $i^{\prime}(x, y)$ is achieved using (6) and (7) and it is shown as follows,

$$
I^{\prime}(f, \theta)=s \cdot I\left(\frac{f}{s}, \theta\right)
$$

As $p^{\prime}(\theta)$ integrates $B(f, \theta)$ between 0 and a half of the Nyquist frequency, assuming $\frac{f}{s}<0.5$, (11) will show the same result with that of using $I(f, \theta)$. In practice, we found this assumption can be accepted up to $50 \%$ scaling down. This can be shown as follows,

$$
\begin{aligned}
p^{\prime}(\theta) & =L\left[\int_{0^{+}}^{0.5} s^{3} I^{2}\left(\frac{f}{s}, \theta\right) \cdot I^{*}\left(\frac{2 f}{s}, \theta\right) d f\right] \\
& =L\left[\int_{0^{+}}^{0.5 / s} I^{2}\left(\frac{f}{s}, \theta\right) \cdot I^{*}\left(\frac{2 f}{s}, \theta\right) d f\right] \\
& =L\left[\int_{0^{+}}^{0.5} I^{2}(f, \theta) \cdot I^{*}(2 f, \theta) d f\right] \\
& =p(\theta)
\end{aligned}
$$

As $p(\theta)$ is invariant to scale, $\boldsymbol{p}$ is invariant to scale.

\subsection{Rotation}

An image $i^{\prime}(x, y)$ rotated by $\alpha^{\circ}$ and its Fourier transform can be expressed as,

$$
\begin{aligned}
i^{\prime}(x, y) & =i(x \cos \alpha+y \sin \alpha,-x \sin \alpha+y \sin \alpha) \\
I^{\prime}\left(f_{x}, f_{y}\right) & =I\left(f_{x} \cos \alpha+f_{y} \sin \alpha,-f_{x} \sin \alpha+f_{y} \cos \alpha\right)
\end{aligned}
$$

The polar mapped $I^{\prime}(f, \theta)$ will have circularly shifted $I(f, \theta-\alpha)$ as 2-D Fourier transform rotates as well when an image rotates. This can be shown from $I^{\prime}\left(f_{x}, f_{y}\right)$ substituting (13) with (6) and (7) as follows, 


$$
\begin{aligned}
I^{\prime}\left(f_{x}, f_{y}\right) & =I(f \cos \theta \cos \alpha+f \sin \theta \sin \alpha,-f \cos \theta \sin \alpha+f \sin \theta \cos \alpha) \\
& =I(f \cos (\theta-\alpha), f \sin (\theta-\alpha)) \\
I^{\prime}(f, \theta) & =I(f, \theta-\alpha)
\end{aligned}
$$

Then, $p^{\prime}(\theta)$ becomes,

$$
\begin{aligned}
p^{\prime}(\theta) & =L\left[\int_{0^{+}}^{0.5} I^{2}(f, \theta-\alpha) I^{*}(2 f, \theta-\alpha) d f\right] \\
& =p(\theta-\alpha)
\end{aligned}
$$

Hence, $\boldsymbol{p}$ will be circularly shifted according to the rotation by $\alpha^{\circ}$ and for the detection, we will circularly shift $\boldsymbol{p}$ before measuring similarity.

\section{Algorithm}

We use an invariant feature vector of the image as a watermark. The watermark is embedded by selecting a vector from the set of extracted feature vectors. The chosen feature vector is used as the watermark and the inverted image is used as the watermarked image. The watermarks are generated through an iterative feature modification and verification procedure. This procedure avoids the interpolation errors that can occur during insertion and detection of the watermark. At detector, the feature vector is estimated from the test image. We use rootmean-square-error (RMSE) as our similarity measure instead of the traditional normalized correlation. It is because the feature vectors are not white and the correlation measure can not produce peak value when they are same vectors. Hence, we measure the distance between two vectors using RMSE function. If the RMSE value is smaller than the threshold, the watermark is detected. The original image is not required at the detector. We define the detector first and an iterative embedder is designed using the detector.

\subsection{Watermark Detection}

Detector takes a test image $I(x, y)$ and extracts a feature vector $\boldsymbol{p}$ of length $N$ from the polar mapping of the Fourier spectrum of $I(x, y)$. The similarity $s$, is defined with RMSE between the extracted vector $\boldsymbol{p}$ and the watermark $s$ as following,

$$
s(\boldsymbol{p}, \boldsymbol{w})=\sqrt{\frac{1}{N} \sum_{i=1}^{N}\left[p\left(\theta_{i}\right)-w_{i}\right]^{2}}
$$

where $N$ is the length of the feature vector. If $s$ is smaller than the detection threshold $T$, the watermark is detected. 


\subsection{Watermark Embedding}

A watermark is embedded by modifying the feature vector $\boldsymbol{p}$ of an input image. The 2-D Fourier transform $X\left(f_{1}, f_{2}\right)$ of an $M \times M$ input image $I$ is computed and polar mapped to construct the $M \times N$ 2-D matrix $X_{p}(f, \theta)$ at $N$ evenly spaced angles from 0 to $180^{\circ}$. We can shape the feature vector $\boldsymbol{p}$ by modifying some of its components $p\left(\theta_{w}\right)$ at $\theta_{w} \in 0, \ldots, 180$. If we shift all the phase spectrum of the image by $\delta$ along the radial angle of $\theta_{w}$, we have a modified component $p^{\prime}\left(\theta_{w}\right)$ as follows:

$$
\begin{aligned}
p^{\prime}\left(\theta_{w}\right) & =L\left[\int_{0^{+}}^{0.5} X(f) e^{j \delta} X(f) e^{j \delta} X^{*}(f+f) e^{-j \delta} d f\right] \\
& =L\left[\int_{0^{+}}^{0.5} X(f) X(f) X^{*}(f+f) d f\right]+\delta \\
& =p\left(\theta_{w}\right)+\delta
\end{aligned}
$$

After shifting the phases of the selected columns of $X_{p}(f, \theta)$, we inverse transform it to have the watermarked image $I^{\prime}$.

However, we cannot extract the embedded signal $\delta$ from $I^{\prime}$ at detector. As reported in the previous researches [5][6], algorithms that modify the Fourier coefficients in polar or log-polar domain suffer three problems. First, interpolation at embedder causes errors at detector. During the polar or log-polar mapping, an interpolation method should be involved because we are dealing with discrete image data. Though we choose more accurate interpolation function, there will be some errors as long as we are working with discrete images. Second, zeropadding at detector degrades the embedded signal further. By zero-padding, spectrum resolution is improved but interpolation error is increased. Third, the interrelations of the Fourier coefficients in the neighboring angles cause 'smearing' effect of the modified feature values. If we modify a single component $p(\theta)$, it affects other values nearby.

In [7], the authors have provided approximation methods to reduce the effects of these errors. Instead of using a similar method, we approach this problem differently. After modifying some components of the feature vector, the watermarked image which contains the implementation errors is produced by the inverse 2-D DFT. We extract the feature vector from this watermarked image and use it as the embedded watermark instead of the initially modified feature vector. In this way, we can embed the watermark without exact inversion of the modified signal.

However, to guarantee the uniqueness of the watermark and its perceptual invisibility after insertion, we need a verification procedure to use it as a watermark. Fig. 2 shows the geometry of feature vector space.

Black dots represent feature vectors and each dot corresponds to a feature vector of an image. The solid circles show the robustness boundaries resulted from geometric distortion. $r 1$ is the maximum distortion of the feature vector resulted from a geometric distortion. The inside region of the solid circle means 


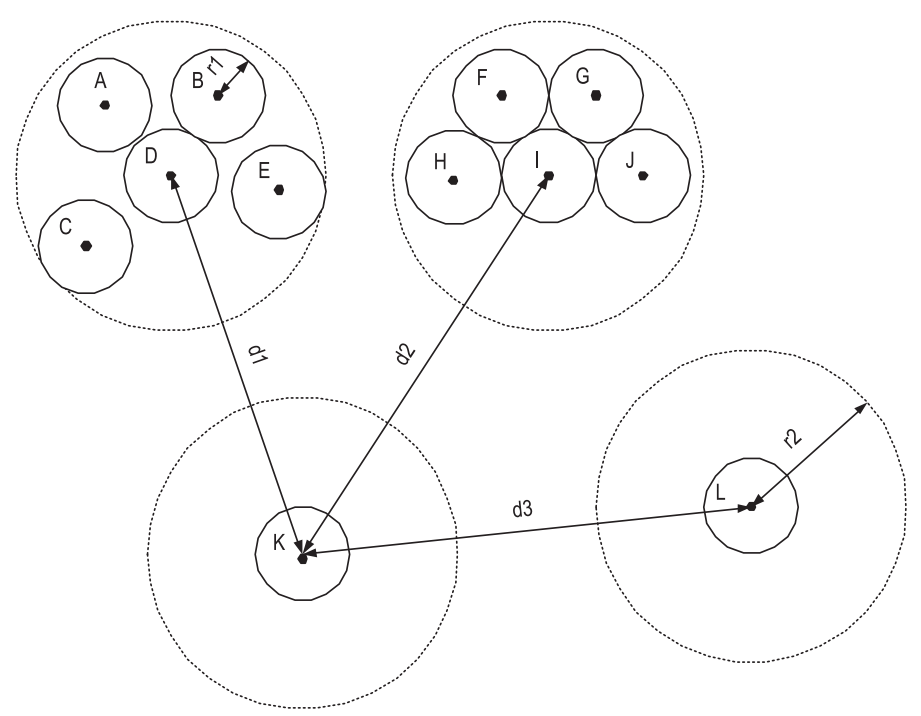

Fig. 2. Geometrical modeling of feature vector space

a set of distorted images where the centered feature vector can be successfully extracted. The dotted circles show the minimum feature distance $r 2$ between the feature vectors of unmarked images. The distance $r 2$ is determined empirically by measuring the feature distances $d 1, d 2, d 3, \ldots$ between unmarked images and taking the minimum among them. The feature distances $r 1$ and $r 2$ are measured with the similarity $s$. Vectors D, I, K, and L are the feature vectors extracted from four different unmarked images. Vectors A, B, C, and E are the valid watermarks modified from a feature vector D. For a modified feature vector to be used as a valid watermark, the distance between the modified vector and original vector should be larger than $r 1$ and smaller than $r 2$. During embedding, the extracted feature vectors from the inverted image will be checked if they meet the requirements for valid watermarks. This validity check routine will be performed iteratively until we get the distinguishable $s$ and meet unobtrusiveness requirement.

\section{Experimental Results}

Experiments are performed with 100 images from the Corel image library [17]. For valid watermark generation, $r 1$ and $r 2$ are determined empirically using unwatermarked images. The similarity $s$ is measured between unmarked test images and the smallest $s$ is chosen for $r 2$. For the determination of $r 1$, robustness of the defined feature vector is tested. Similarity $s$ is measured between the original image and attacked images. The largest $s$ is chosen for $r 1$. For the robustness test, we set $r 1=4.5$ and $r 2=20$. Feature vectors are modified with $\delta=5^{\circ} \sim 7^{\circ}$ at randomly selected angles. The number of insertion angles 


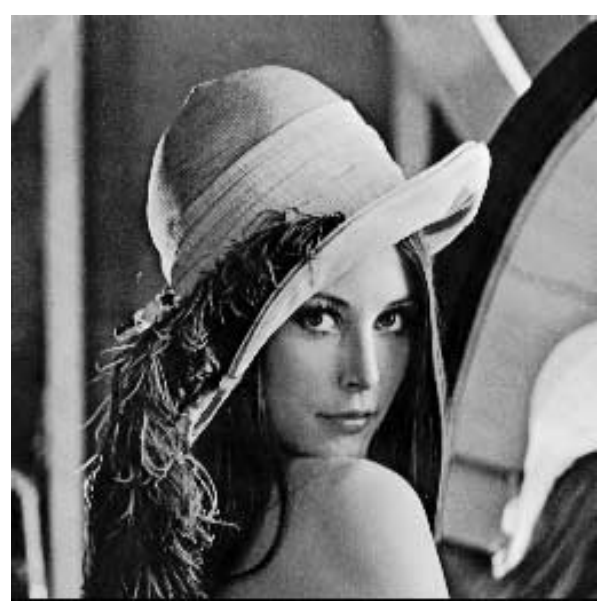

(a)

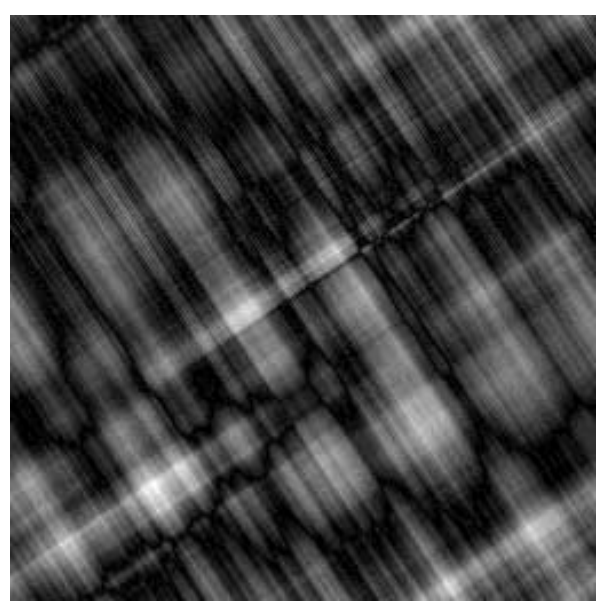

(b)

Fig. 3. Embedding example : (a) Watermarked Lena image embedding at $\theta_{w}=$ $35^{\circ}$ and $125^{\circ}$ (b) Amplified difference between wateraraked and original image

is randomly determined between 1 and 3. $T=4.5$ is used for the detection threshold. Watermarks are generated using the iterative procedure described in section 3.2. During the iteration, parameters are adjusted accordingly. Fig. 3 shows the watermarked Lena image and the amplified difference between original and watermarked images.

The watermarked image shows PSNR of $36 d B$ and the embedded signal is invisible. During the watermark insertion, we maintained the PSNR higher than $36 d B$. Robustness of the watermark against each attack is measured with 100 unmarked images and 100 marked images. We measure the empirical probability density function (pdf) of the computed with histogram. Assuming that the empirical pdf of $s$ can be approximated by a normal distribution, false positive probability $\left(P_{f p}\right)$ and false negative probability $\left(P_{f n}\right)$ can be computed using the estimates of mean and variance. Random geometric attack performance is the worst with $P_{f p}=4.00 \times 10^{-3}$ and $P_{f n}=6.20 \times 10^{-2}$. It shows that our method performs well over the intended attacks. The similarity histograms and receiver operating characteristic (ROC) curves $\left(P_{f p}\right.$ versus $P_{f n}$ for several thresholds) are produced for analysis. In this section, five attacks are examined: rotation, scaling, random geometric distortion, compression and Gaussian noise.

\subsection{Rotation}

Fig. 4 shows the histogram of s and ROC curve. Though the rotation by large angle can be detected by cyclically shifting the extracted feature vector, the performance of rotation by a large angle is poor due to the difficulty of interpolation in the Fourier phase spectrum. For this reason, we show the results of rotation by small angles. With $T=4.5, P_{f p}$ is $6.03 \times 10^{-2}$ and $P_{f n}$ is $1.90 \times 10^{-3}$. False 


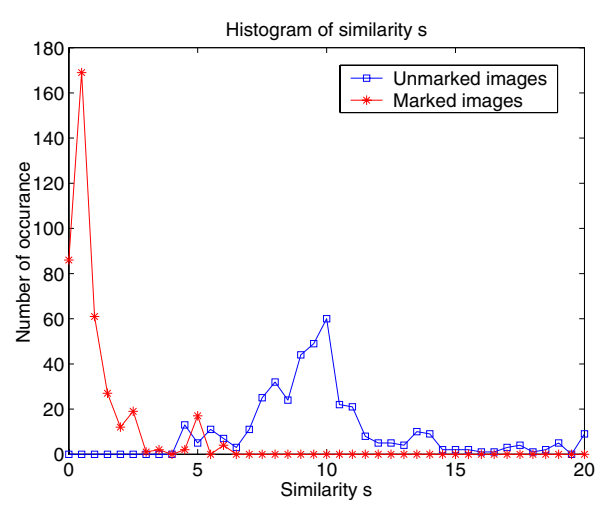

(a)

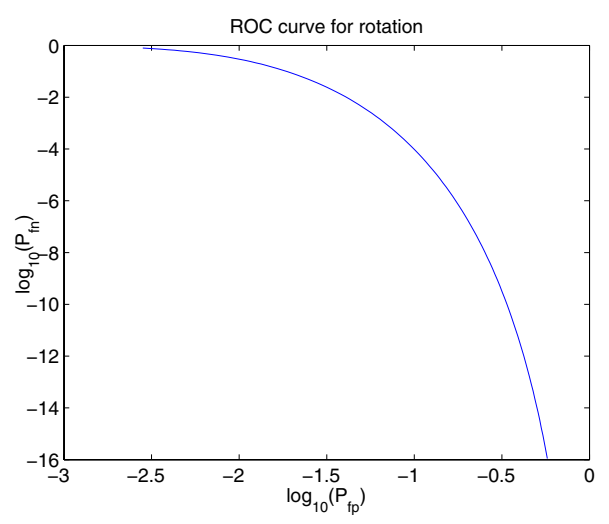

(b)

Fig. 4. Rotation $\pm 0.25^{\circ}, \pm 0.5^{\circ}$ : (a) Histogram of $s$ (b) ROC Curve

negative probability shows better performance than false positive probability in this attack. This is because the pdf of the similarity between unmarked images and watermarks has relatively large variance that resulted into the larger false positive probability. As $P_{f p}$ and $P_{f n}$ show, our method is robust against rotation by small angle.

\subsection{Scaling}

The detection histogram was measured using 50\% scaled down images and $200 \%$ scaled up images. As the histogram in Fig. 5 shows, the watermarked images show strong resistance to scaling attack. The ROC curve shows that $P_{f p}$ is $5.6 \times 10^{-3}$ and $P_{f n}$ is $1.14 \times 10^{-4}$. These values are relatively lower than other attacks and this means our method performs well with scaling attacks. Our method has strong robustness against scaling attack even after scaling down to $50 \%$.

\subsection{Random Geometric Distortion}

This attack simulates the print-and-scanning process of images. It applies a minor geometric distortion by an unnoticeable random amount in stretching, shearing, and/or rotating an image [2]. In Fig. 6, the histogram shows large variance in the similarity between watermark and unmarked image. As the result, $P_{f p}$ is $4.0 \times 10^{-3}$ and $P_{f n}$ is $6.2 \times 10^{-2}$, which are relatively large compared with others. Not many previous methods survive this attack and our algorithm works well even with those numbers.

\subsection{Compression}

JPEG compression with $\mathrm{Q}=30$ and 70 was applied after watermark embedding. With $\mathrm{Q}=30$, the watermarked image fidelity is unacceptable. However, our method survives the harsh compression attack. Fig. 7 shows the histogram 


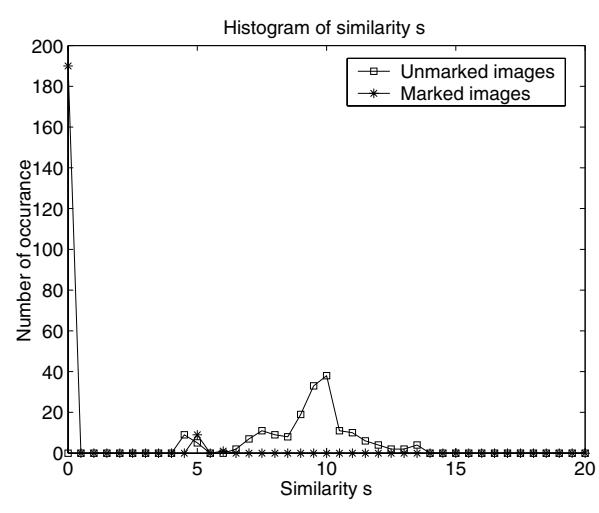

(a)

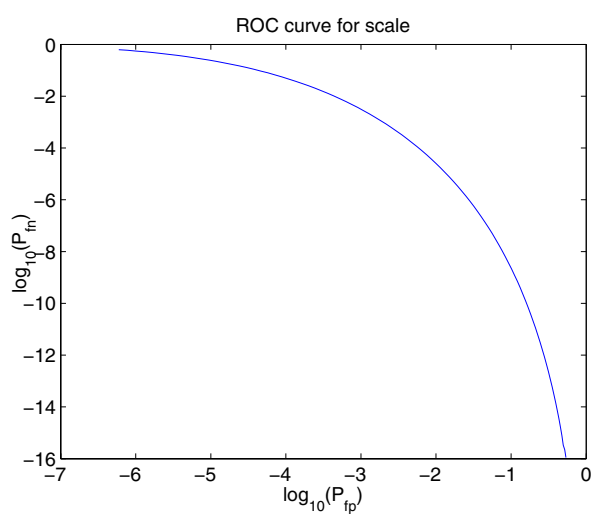

(b)

Fig. 5. Scale 50\%, 200\%: (a) Histogram of $s$ (b) ROC Curve

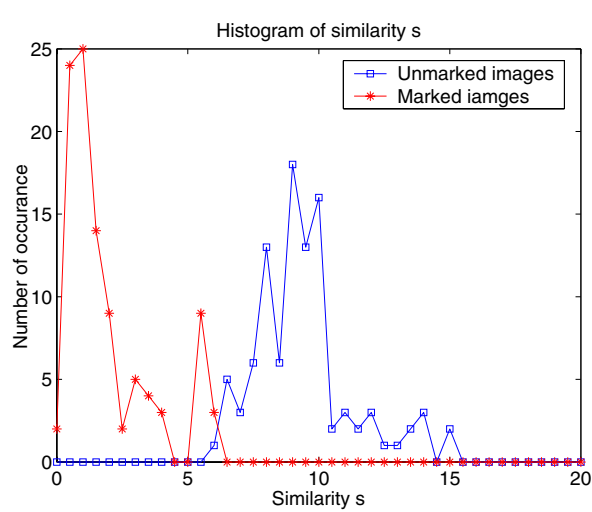

(a)

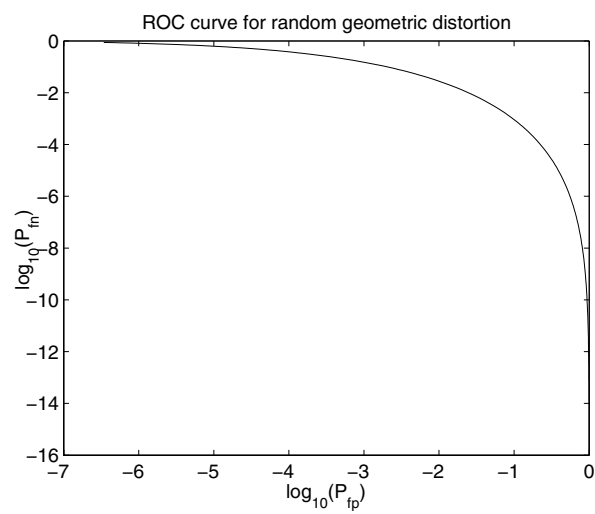

(b)

Fig. 6. Random geometric distortion : (a) Histogram of $s$ (b) ROC Curve

and ROC curve. $P_{f p}$ is $3.5 \times 10^{-3}$ and $P_{f n}$ is $2.2 \times 10^{-20}$. The false negative probability is extremely low and this is because our feature vector is not affected by any high frequency noises. Our method has strong resilience to JPEG compression.

\subsection{Gaussian Noise}

As our invariant feature vector is defined from HOS, it must be invariant against additive Gaussian noise. Gaussian noise was added to the watermarked image by convolving a $3 \times 3$ kernel as follows 


$$
\mathbf{G}=\left[\begin{array}{lll}
1 & 2 & 1 \\
2 & 4 & 2 \\
1 & 2 & 1
\end{array}\right]
$$

The histogram of the similarity of unmarked and marked images is shown in Fig. 12. (a). The ROC curve is shown in Fig. 8. From the curve, $P_{f p}$ is $6.78 \times 10^{-4}$ and $P_{f n}$ is $1.9 \times 10^{-20}$. These probabilities show that our method is robust against the Gaussian noise.

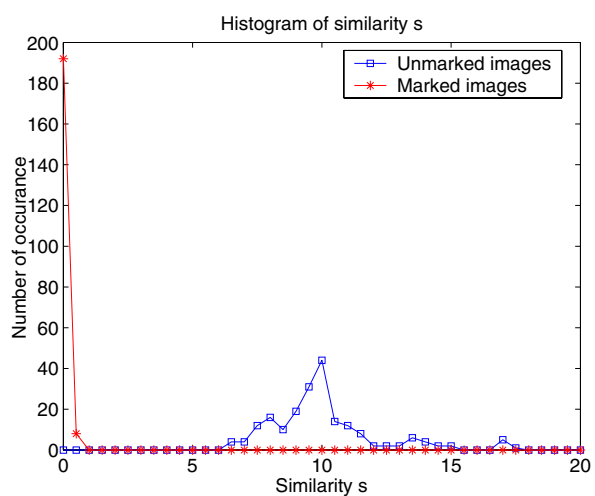

(a)

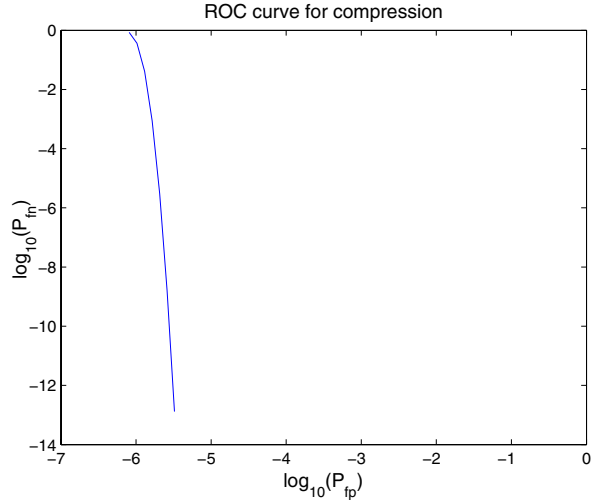

(b)

Fig. 7. JPEG Compression $\mathrm{Q}=30$ and 70 : (a) Histogram of $s$ (b) ROC Curve

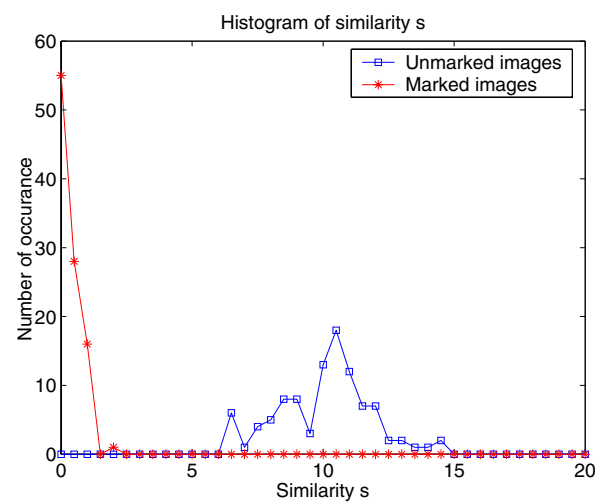

(a)

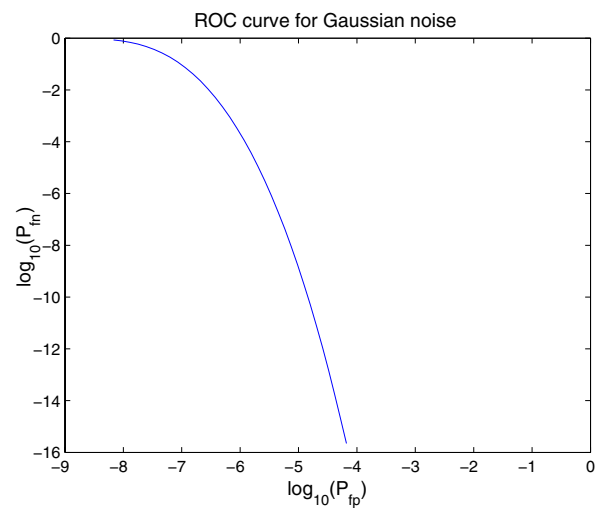

(b)

Fig. 8. Additive Gaussian noise : (a) Histogram of $s$ (b) ROC Curve 


\section{Conclusions}

We propose a new RST invariant watermarking method based on an invariant feature of the image. A bispectrum feature vector is used as the watermark and this watermark has a strong resilience on RST attacks. This approach shows a potential in using a feature vector as a watermark. An iterative informed embedding procedure is designed to overcome the problem of inverting watermarked image. This method can be generalized for other embedding functions that do not have exact inverse function.

In all our experiments, we have shown the empirical probability density functions with histograms and the ROC curves. Experimental results show that our scheme is robust against wide range of attacks including rotation, scaling, JPEG compression, random geometric distortion and Gaussian noise.

\section{Acknowledgements}

This work was supported by the Korea Science and Engineering Foundation (KOSEF) through the Advanced Information Technology Research Center (AITrc). The authors would like to thank Dr. Ingemar Cox and Matt Miller of NEC Research Institute for helpful discussions on Fourier-Mellin transform and watermarking with side information. In addition, the authors would like to thank K. M. Park of Korea Advanced Institute of Science and Technology (KAIST) for helpful discussions on higher order spectra.

\section{References}

[1] F. Hartung and M. Kutter, "Multimedia watermarking technique," Proc. IEEE, Vol 87, pp. 1079 1107, July, 1999. 145

[2] F. A. P. Petitcolas, R. J. Anderson, and M. G. Kuhn, "Attacks on copyright marking systems," in Proc. 2nd Int. Workshop on Information Hiding, pp. 218-238, 1998. 145, 147, 155

[3] S. Pereira and T. Pun, "Robust template matching for affine resistant image watermarksI," IEEE Trans. Image Processing, Vol 9, pp. 1123-1129, July, 2000. 146

[4] G. Csurka, F. Deguillaume, J. J. K. O'Ruanaidh, and T. Pun, "A Bayesian approach to affine transformation resistant image and video watermarking, " Proc. 3rd Int. Workshop on Information Hiding pp. 315-330, 1999. 146

[5] M. Kutter, "Watermarking resisting to translation, rotation, and scaling," Proc. SPIE Multimedia Systems Applications, pp. 3528 423-431, 1998. 146, 152

[6] J. J. K. O'Ruanaidh and T. Pun, "Rotation, scale, and translation invariant spread spectrum digital image watermarking", Signal Processing, Vol 66, pp. 303-317, 1998. 146, 147, 152

[7] C. Y. Lin, M. Wu, J. A. Bloom, I. J. Cox, M. L. Miller and Y. M. Lui, "Rotation, scale, and translation resilient watermarking for images," IEEE Trans. Image Processing, Vol 10. May, pp. 767-782, 2001. 146, 147, 152 
[8] M. Kutter, S. K. Bhattacharjee, and T. Ebrahimi, "Towards second generation watermarking schemes," Proc. IEEE Int. Conf. Image Processing, pp. 320-323, 1999. 146

[9] S. Guoxiang and W. Weiwei, "Image-feature based second generation watermarking in wavelet domain," Lecture Notes in Computer Science, Vol 12251, pp. 16-21, 2001. 146

[10] C. Nikias and M. Raghuveer, "Bispectrum estimation: A digital signal processing framework," Proc. IEEE, Vol 75, July pp. 869-889 1987. 146

[11] C. L. Nikias and A.P. Petropulu, Higher-Order Spectra Analysis, PTR Prentice Hall, Englewood Cliffs, New Jersey, 1993. 146

[12] V. Chandran, B. Carswell, B. Boashash, and S. Elgar, "Pattern recognition using invariants defined from higher order spectra: 2-D image inputs," IEEE Trans. Image Processing, Vol 6, May, pp. 703-712, 1997. 146, 149

[13] Y. Horikawa, "Bispectrum-based feature of 2-D and 3-D images invariant to similarity transformations," Proc. IEEE Int. Conf. Pattern Recognition, pp. 511-514, 2000. 146

[14] I. J. Cox, M. L. Miller, and A.L. Mckellips, "Watermarking as communications with side information", Proc. IEEE, Vol 87, July, pp. 1127-1141, 1999. 147

[15] M. H. Hayes, "The reconstruction of a multidimensional sequence," IEEE Trans. Acoustics, Speech and Signal Processing, Vol 4, pp. 140-154, 1992. 147

[16] A. K. Jain, Fundamentals of Digital Image Processing, Prentice Hall, Englewood Cliffs, NJ, 1989. 148

[17] Corel Corporation, Corel Stock Photo Library 3. 153 